\title{
Quantification of Myocardial Enhancement on Cine-MRI: Diagnostic Value in Cardiac Amyloidosis
}

Clément Cholet ${ }^{1} \mathrm{MD}$; clementcholet@gmail.com

Thibaud Damy ${ }^{2,3,4,5}$ MD, PhD; thibaud.damy@aphp.fr

François Legou ${ }^{1}$ MD; francois.legou@aphp.fr

Hicham Kobeiter ${ }^{1,3,5}$ MD, PhD; hicham.kobeiter@aphp.fr

Alain Rahmouni ${ }^{1,3} \mathrm{MD}, \mathrm{PhD}$; alain.rahmouni@aphp.fr

Jean-François Deux ${ }^{1,3,4,5}$ MD, PhD; jean-francois.deux @aphp.fr

${ }^{1}$ Assistance Publique - Hôpitaux de Paris (AP-HP), Service d'Imagerie Médicale, CHU Henri Mondor, 51 avenue du Maréchal de Lattre de Tassigny, Créteil, 94010, France.

${ }^{2}$ Assistance Publique - Hôpitaux de Paris (AP-HP), Service de Cardiologie, CHU Henri Mondor, 51 avenue du Maréchal de Lattre de Tassigny, Créteil, 94010, France.

${ }^{3}$ Université Paris-Est Créteil (UPEC), Créteil, 94010, France.

${ }^{4}$ GRC Amyloid Research Institute and Réseau Amylose Mondor, CHU Henri Mondor, UPEC, Créteil, 94010, France.

${ }^{5}$ DHU, ATVB, UPEC, Créteil, 94010, France.

\section{Corresponding Author:}

Clément Cholet, MD

Address: CHU Henri Mondor, Service d'Imagerie Médicale, 51 avenue du Maréchal de Lattre de Tassigny, Créteil, 94010, France.

Telephone: (+ 33) $149812631 /(+33) 684893795$

Fax: (+33) 149812632

E-mail address: clementcholet@gmail.com

ORCID number: 0000-0002-1370-1514

Declaration of interest: None.

Funding: There was no funding secured for this study. 


\section{Quantification of Myocardial Enhancement on Cine-MRI: Diagnostic Value in Cardiac}

\section{Amyloidosis}

\section{ABSTRACT \\ Rationale and Objectives}

Diagnosis of cardiac amyloidosis $(\mathrm{CA})$ on cardiac magnetic resonance $(\mathrm{CMR})$ can be challenging and quantitative indexes are relevant to further characterize the myocardium. We hypothesize that the relative myocardial enhancement measured from pre and post contrast cine imaging provides diagnostic information for $\mathrm{CA}$ in the setting of left ventricular hypertrophy (LVH).

\section{Materials and Methods}

Patients with LVH referred to our center and control subjects with normal CMR were retrospectively included. Percentage of myocardial enhancement (percentage ME) was obtained from pre and post contrast (5 minutes) cine sequences. Post contrast myocardial T1 and LGE extent were also recorded.

\section{Results}

Twenty-one patients with CA, 25 patients with non-amyloid left ventricular myocardial hypertrophy $(\mathrm{CH})$ and 20 controls with normal CMR were analyzed. Percentage ME was significantly higher in CA patients $(200 \%$ (174-238)) than in $\mathrm{CH}$ patients $(122 \%(88-151)$; $\mathrm{p}=0.0001)$ and control patients $(104 \%(90-149) ; \mathrm{p}=0.0001)$. Percentage ME was significantly correlated with the LGE extent (Rho Spearman coefficient $=0.66 ; \mathrm{p}=0.0001$ ) and with the post contrast myocardial T1 (Rho Spearman coefficient $=-0.61 ; \mathrm{p}=0.0001$ ). With a cutoff value of $152 \%$, the sensitivity and specificity of percentage ME for detection of CA were $90 \%$ and $80 \%$, respectively.

\section{Conclusion}


Percentage ME obtained from pre and post contrast cine imaging is correlated to LGE extent and myocardial T1 and may represent an additional diagnostic parameter to consider CA in patients with LVH.

\section{KEYWORDS}

Cardiac Magnetic Resonance; Amyloidosis; Balanced-Steady State Free Precession; Diagnostic Test. 


\section{INTRODUCTION}

Cardiac amyloidosis (CA) is a restrictive cardiomyopathy, classically responsible for left ventricular myocardial hypertrophy $(\mathrm{LVH})$ and preserved left ventricular ejection fraction (LVEF) [1]. Although often overlooked, CA is more frequent than expected, and groups have reported that up to $5 \%$ of patients presenting with $\mathrm{LVH}$ have amyloid cardiomyopathy [2]. Early identification of CA in the setting of myocardial hypertrophy is fundamental because [3]: i) early introduction of specific anti-amyloid drugs ameliorates survival; ii) classical management of heart failure can be deleterious in these patients (including the use of betablockers); and iii) late detection of CA is associated with a worse prognosis.

Cardiac magnetic resonance imaging (CMR) plays a central role in the diagnosis of CA. Typical aspect of CA on CMR includes left ventricular (LV) wall thickening, subendocardial or diffuse myocardial enhancement on late gadolinium enhancement (LGE) imaging, and abnormal gadolinium kinetics in the myocardium and the blood pool [4,5]. Despite these typical features, diagnosis with CMR remains challenging as patterns of hypertrophy and LGE can sometimes be misleading [6,7]. Furthermore, LGE imaging can be difficult to perform in CA because of amyloid deposit leading to suboptimal nulling of the myocardium, particularly when using TI scouting techniques [8]. Therefore, quantitative index are relevant, especially in case of equivocal CMR or to quantify the amyloid burden, a major marker of outcome in systemic amyloidosis $[9,10]$. Many authors have reported that mapping sequences could provide quantitative parameters such as myocardial $\mathrm{T} 1$ and extra-cellular volume (ECV) measurements. These parameters increased diagnostic accuracy for CA and could be helpful to quantify the amyloid burden [11-13]. Unfortunately, mapping sequences are not yet widely available, normal values of myocardial $\mathrm{T} 1$ can change between different types of sequences and are influenced by physiological parameters [14,15]. 
Balanced steady-state free precession (b-SSFP) cine sequences are routinely used in standard CMR scanning [16]. They are robust, provide an excellent contrast between the blood pool and the myocardium and are usually of good quality [17]. They are commonly used without contrast to assess the global and regional cardiac function. After gadolinium administration, and because of the dual T2/T1 component of their signal, they are sensitive to post-contrast myocardial T1 shortening, and can evidence focal enhancement in cases of myocarditis or recent myocardial infarction [18,19]. More recently, they appeared useful in identifying myocardium at risk in ST-elevation myocardial infarction [20]. However, these sequences have never been reported to detect a diffuse myocardial disease such as CA. In this study, we hypothesize that the abnormal uptake of gadolinium in CA increases the signal intensity (SI) of the LV myocardium on post contrast cine images and that these sequences may provide additional support for $\mathrm{CA}$ in clinical practice. Thus, we measured the relative level of myocardial enhancement using pre and post contrast cine images in patients with CA, patients with non-amyloid myocardial hypertrophy $(\mathrm{CH})$, and patients with normal CMR. We assessed the diagnostic performance of post contrast cine-MRI to detect CA and compared it with other quantitative imaging markers including LGE extent, post contrast myocardial T1 and myocardial contraction fraction (MCF).

\section{METHODS}

We performed a retrospective monocentric study at our institution. Informed consent was obtained for every patient, and the institutional review board of our hospital approved the study.

Study Population 
We retrospectively included patients from the register of the reference center for amyloidosis at our institution. Patients were addressed to this network for therapeutic management of proven CA or diagnostic assessment of LVH. All patients included had been admitted to our center between March 2011 and October 2013 and presented LVH defined by end-diastolic myocardial wall thickness $\geq 12 \mathrm{~mm}$ on TTE or CMR involving at least 1 segment. We excluded patients when: i) CMR was not performed in our center; ii) sequence parameters were not identical between pre and post-contrast cine-MRI; and iii) the cause of myocardial hypertrophy was uncertain. After review of their electronic medical record, patients were separated into 2 groups according to the cause of myocardial hypertrophy.

The CA group was composed of patients with proven transthyretin-related (ATTR) amyloidosis (hereditary ATTR or systemic senile amyloidosis) or light chain (AL) amyloidosis. Endomyocardial biopsy (EMB) confirming CA was obtained for all AL patients. For TTR patients, diagnosis of cardiac involvement was made on significant heart retention of 99mTc-HMDP on scintigraphy [21].

The non-amyloid $\mathrm{CH}$ group consisted of patients with $\mathrm{LVH}$ not related to amyloidosis. The cause of hypertrophy was obtained through review of electronic medical records for the respective patient. Final diagnosis had been established by the cardiology team on a body of clinical, electrical, biological and imaging arguments.

Finally, a control group of subjects with normal CMR was included. These subjects were obtained from a register proper to the radiology department of our institution. Normal CMR study was defined as follows: normal LV myocardial thickness (defined a wall thickness $<12$ $\mathrm{mm}$ on end-diastolic short axis cine images), normal LVEF (>50\%), normal left ventricular end-diastolic volume (LVEDV) and left ventricular end-systolic volume (LVESV), absence of segmental contraction impairment, absence of focal or diffuse myocardial edema on STIR T2 images and absence of LGE. All demonstrated normal right ventricular function. 


\section{Biological and TTE data}

NT-pro BNP (pg/mL), troponin T $(\mathrm{pg} / \mathrm{mL})$ and creatinine levels $(\mu \mathrm{mol} / \mathrm{L})$ were obtained for all patients of the 3 groups. Data from previous transthoracic echocardiography (TTE) were reported for $\mathrm{CA}$ and $\mathrm{CH}$ patients, including end-diastolic interventricular septum (IVS) thickness (mm), global longitudinal strain (-\%) and LVEF (\%). Same data from TTE were recorded for control patients except for global longitudinal strain which was not available.

\section{Cardiac MRI Protocol}

All patients included were imaged using the same protocol with a 1.5 Tesla (T) MRI (Avanto; Siemens Medical; Erlangen; Germany) equipped with a high-performance gradient sub-system (maximum amplitude, $40 \mathrm{mT} / \mathrm{m}$; minimum rise, $200 \mu \mathrm{s}$ ) and an 8-channel phased-array cardiac coil. Gradient echo localizers were used to obtain anatomic axes of the heart. A standard pre-contrast cine imaging was performed to calculate the LVEF, using contiguous short-axis (SA) b-SSFP sections of $8 \mathrm{~mm}$ thickness covering the entire LV. A corresponding mid-ventricular post-contrast cine sequence was systematically performed, at the same ventricular level than the pre-contrast cine imaging, $5.2 \pm 1$ (4-7) minutes after intravenous administration of a bolus of $0.2 \mathrm{mmol} / \mathrm{kg}$ body weight of gadolinium chelate (gadoterate dimeglumine; Dotarem; Guerbet; France), injected at a rate of $3.5 \mathrm{~mL} / \mathrm{s}$, followed by a 30-mL saline flush. The following parameters were used both for pre- and post-contrast SSFP cine sequences: TR/TE, 30.8/1.4; flip angle, 82; matrix size, 192 x 192; FOV, 300 x $270 \mathrm{~mm}$; slice thickness, $8 \mathrm{~mm}$. Retrospective ECG gating was used with 20 phases per section. LGE imaging was acquired 10 minutes after contrast administration, in the fourchamber, vertical long-axis, and SA planes using a segmented 3D inversion recovery gradient-echo T1-weighted technique, following a TI scout sequence. Acquisition was 
performed during end-diastole. LGE imaging parameters were as follows: TR/TE, 3.9/1.4; mean inversion recovery time, 220 +/- $50 \mathrm{~ms}$; flip angle, 10² matrix size, 192 x 192; FOV, 300 x $270 \mathrm{~mm}$; number of sections, 12; slice thickness, $6 \mathrm{~mm}$. Because suboptimal nulling of the myocardial signal may be encountered in CA, phase sensitive inversion recovery (PSIR) images were systematically acquired after acquisition. Parameters of PSIR sequences were as follows: TR/TE, 835/3.3 ms; flip angle, 10; matrix size, 256 × 156; FOV, 300 x 270 mm; number of sections, 5; slice thickness, $8 \mathrm{~mm}$. Images were acquired in the SA plane.

\section{Cine-MRI images analysis}

Cine images analysis was performed on a dedicated workstation (Leonardo; Siemens Healthcare; Germany) by one operator with 3-year experience in cardiac imaging, after anonymization of patients and groups. IVS thickness was measured on a pre-contrast cine image acquired in the SA plane, at the mid-LV level in end-diastole. LVEDV, LVESV, LV mass and LVEF were obtained from pre contrast cine imaging using a dedicated software (Argus; Siemens Medical System; Germany). LV myocardial SI was measured using a region of interest (ROI) set manually to encompass the whole myocardium on SA cine images at the mid-LV level, in end-diastole. The process was repeated for the corresponding post contrast cine images. ROIs were drawn to avoid contamination from blood and pericardial fat. A 1 $\mathrm{cm}^{2}$ ROI was placed in the background air on the corresponding pre and post contrast SA cine images in order to measure standard deviation (SD) of the SI of the air ( $\left.\mathrm{SD}_{\text {air }}\right)$.

To quantify the level of myocardial enhancement on cine b-SSFP sequences, we introduced a new value, the percentage of myocardial enhancement (percentage ME), defined as the difference in SI between pre and post contrast cine images and normalized for the SI of the pre contrast cine image. Percentage ME was calculated using the following formula:

$$
\text { Percentage } M E(\%)=\frac{S I_{\text {myocardium post contrast }}-S I_{\text {myocardium pre contrast }}}{S I_{\text {myocardium pre contrast }}} \times 100
$$


Intra and inter-examiner reproducibility of the percentage ME was checked by Bland-Altman plots on a panel of subjects from each group, after blinded double reading by 2 radiologists. Signal-to-noise ratio (SNR) of the myocardium was calculated on pre and post contrast cine images using the following formula:

$$
S N R_{\text {myo }}=\frac{S I_{\text {myo }}}{S D_{\text {air }}}
$$

Contrast-to-noise ratio (CNR) of the myocardium was also calculated on pre and post contrast cine images using the following formula:

$$
C N R_{\text {myo }}=\frac{S I_{\text {myo }}-S I_{\text {blood }}}{S D_{\text {air }}}
$$

Finally, the myocardial contraction fraction (MCF) was calculated as recently reported [22]:

$$
\operatorname{MCF}(\%)=\frac{L V \text { stroke volume }(\mathrm{mL})}{L V \text { myocardial volume }(\mathrm{mL})} \times 100
$$

where $L V$ stroke volume $=\mathrm{LVEDV}-\mathrm{LVESV}$ and $L V$ myocardial volume $=\mathrm{LV}$ myocardial mass / mean density of myocardium $(1.05 \mathrm{~g} / \mathrm{mL})$.

\section{Post-contrast myocardial T1}

Post contrast myocardial T1 (ms) was calculated for all patients from the TI scout sequence using a dedicated software (CVi42; Circle Cardiovascular Imaging; Calgary; Canada) as previously described [23]. A ROI was manually drawn to encompass the whole LV myocardium and automatically propagated to all images of the sequence. If necessary, manual corrections were performed to adjust the position of the ROI on the different phases. Post contrast myocardial T1 was automatically obtained from the software. 


\section{LGE quantification}

LGE of the LV was quantified for all patients using a dedicated software (CVi42; Circle Cardiovascular Imaging; Calgary; Canada). After having manually set endocardial and epicardial contours, a threshold of 5 SD was set to detect pixels presenting a SI above 5 SD of the average signal in the pre-defined "normal" myocardium. Manual LGE adjudication was also performed. Both methods were completed on a single mid-LV PSIR image corresponding to the selected SA cine-MRI image [24]. The result was expressed as LGE extent $(\%)$.

\section{Statistical Analysis}

Quantitative data are expressed as means \pm standard deviation or median (interquartile) depending of normal or non-normal distribution (evaluated with Kolomogorov Smirnov and Kurtosis tests). Numbers and percentages are given for categorical data. For non-normal distribution, Kruskal-Wallis and Mann-Whitney tests were used. For parameters with normal distribution, differences were tested using analysis of variance (ANOVA) and post-hoc analysis using Bonferroni correction at $95 \%$ confidence interval. Spearman's correlation coefficient was calculated to assess correlation between variables. Receiver operating characteristic (ROC) analysis, with corresponding measures of statistical uncertainty (i.e., 95\% confidence intervals), was applied to cine MR parameters, percentage LGE, post contrast myocardial T1, MCF and cardiac mass to identify cut-off value for cardiac involvement. The cut-off values were determined using Youden's test. Sensitivity and specificity were calculated using the thresholds previously defined. Data were considered significant if $\mathrm{p}<0.05$. Analyses were performed using SPSS 16.0 software (SPSS Inc., IBM, Chicago, IL). 


\section{RESULTS}

\section{Study Groups}

Sixty-four patients were screened, and 18 were excluded for the following reasons: CMR not performed at our center $(n=7)$, differences in parameters between the pre and post contrast sequences $(n=8)$ and uncertain cause of $\mathrm{LVH}(\mathrm{n}=3)$. A total of 46 patients were included. The group of patients with CA was composed of 21 patients, 16 patients with ATTR amyloidosis (11 hereditary ATTR and 5 systemic senile amyloidosis) and 5 patients with AL amyloidosis. Twenty-five patients with non-amyloid $\mathrm{CH}$ were included. The cause of myocardial hypertrophy was hypertrophic cardiomyopathy $(n=9)$, systemic arterial hypertension $(n=11)$ and aortic stenosis $(n=5)$. Finally, 20 patients with a normal CMR were included in the control group. These subjects had been addressed for various reasons such as atypical chest pain $(n=4)$, suspicion of myocardial hypertrophy $(n=4)$, clinical myocarditis $(n=4)$, clinical suspicion of arrhythmogenic right ventricular dysplasia $(n=4)$, myocardial contusion $(n=2)$, or conductance disorder $(\mathrm{n}=2)$.

\section{Patients characteristics}

Control patients were significantly $(\mathrm{p}=0.001)$ younger $(49 \pm 18$ years $)$ than $\mathrm{CA}(70 \pm 14$ years) and $\mathrm{CH}(62 \pm 14$ years $)$ patients. IVS thickness measured on TTE was similar in CA and $\mathrm{CH}$ patients (respectively, $17 \pm 5 \mathrm{~mm}$ and $18 \pm 9 \mathrm{~mm}, \mathrm{p}=0.6$ ), but was significantly lower $(\mathrm{p}=0.001)$ in control patients $(9 \pm 2 \mathrm{~mm})$. LVEF was preserved and similar in $\mathrm{CA}$ and $\mathrm{CH}$ patients (respectively, $53 \pm 15 \mathrm{~mm}$ and $53 \pm 14 \mathrm{~mm}, \mathrm{p}=0.9$ ). NT-pro BNP, troponin $\mathrm{T}$ and creatinine levels were significantly higher in the CA group than in other groups. All data are reported in Table 1. 
CA patients exhibited significantly $(\mathrm{p}=0.008)$ higher IVS thickness $(19 \pm 4 \mathrm{~mm})$ and cardiac mass $\left(107 \pm 39 \mathrm{~g} / \mathrm{m}^{2}\right)$ than $\mathrm{CH}$ patients $\left(16 \pm 3 \mathrm{~mm}\right.$ and $90 \pm 34 \mathrm{~g} / \mathrm{m}^{2}$, respectively). Control subjects did not exhibit LVH; their IVS thickness $(9 \pm 2 \mathrm{~mm})$ and cardiac mass $(65 \pm 11$ $\mathrm{g} / \mathrm{m}^{2}$ ) was significantly lower than that of $\mathrm{CA}$ and $\mathrm{CH}$ patients.

Quantitative imaging index were significantly different between $\mathrm{CA}$ and $\mathrm{CH}$ patients. Percentage ME was significantly $(\mathrm{p}=0.0001)$ higher in CA $(200 \%(174-238))$ than in $\mathrm{CH}$ (122\% (88-151)) patients and control patients (104\% (90-149)). However, no significant difference $(\mathrm{p}=0.2)$ was observed between $\mathrm{CH}$ patients and control patients. Visual analysis of Bland-Altman plots demonstrated good intra and inter-examiner reproducibility for the percentage ME (see additional files 1 and 2). Examples of cine-MRI images for $\mathrm{CA}, \mathrm{CH}$ and control patients are reported in Figure 1.

Similarly, LGE extent, whether assessed by a semi-automatic or manual method, exhibited a significant $(\mathrm{p}<0.0001)$ increase between CA $(43 \pm 26 \%)$ and $\mathrm{CH}(11 \pm 15 \%)$ patients. Of note, LGE extent was significantly different $(\mathrm{p}<000.1)$ according to the method used for quantification in the CA group (43 $\pm 26 \%$ for the semi-automatic method vs $65 \pm 22 \%$ for the manual method), whereas results were similar $(\mathrm{p}=0.97)$ in the $\mathrm{CH}$ group $(11 \pm 15 \%$ for the semi-automatic method vs $11 \pm 17 \%$ for the manual method). Additional file 3 illustrates these results in more detail. Both MCF and post contrast myocardial T1 were significantly reduced in $\mathrm{CA}$ patients as opposed to $\mathrm{CH}$ patients: $\mathrm{MCF}$ was significantly $(\mathrm{p}=0.007)$ lower in the CA group (33\% (26-52)) than in the $\mathrm{CH}$ group (54\% (44-64)), and post contrast myocardial T1 was significantly $(\mathrm{p}=0.0001)$ lower in the CA group $(271 \mathrm{~ms}(242-340))$ than in the $\mathrm{CH}$ group (439 ms (386-482)). LGE extent and post contrast myocardial T1 were similar between $\mathrm{CH}$ patients and control subjects. In contrast, MCF was significantly $(\mathrm{p}=0.003)$ higher in control subjects $(79 \%(72-96))$ than in $\mathrm{CH}$ patients $(54 \%(44-64))$. 
No significant differences were observed between groups for pre or post contrast SNR and CNR. All data are reported in Table 2.

\section{Correlations}

Percentage ME was significantly correlated with the LGE extent (Rho Spearman coefficient $=0.75 ; \mathrm{p}=0.0001$ ) and with the post contrast myocardial T1 (Rho Spearman coefficient $=-0.61 ; \mathrm{p}=0.0001)$. These results are represented in Figure 2. Percentage ME was also significantly correlated with troponin $\mathrm{T}$ level (Rho Spearman coefficient $=0.44$; $\mathrm{p}=0.006$ ), NT-pro BNP level (Rho Spearman coefficient=0.38; $\mathrm{p}=0.03$ ), LV mass (Rho Spearman coefficient $=0.39 ; \mathrm{p}=0.001$ ), IVS thickness measured on CMR (Rho Spearman coefficient $=0.39 ; \mathrm{p}=0.001)$ and $\mathrm{MCF}$ (Rho Spearman coefficient $=-0.38 ; \mathrm{p}=0.002)$.

\section{ROC curves}

To identify a percentage ME threshold that detects CA, patients with CA formed the positive cases group and $\mathrm{CH}$ patients were considered as negative cases. Control patients were excluded in order to reflect clinical practice. The mean percentage ME exhibited an AUC of 0.92 (CI: 0.84-0.99; p=0.0001) for diagnosed CA patients as opposed to $\mathrm{CH}$ patients (Figure 3). With a cutoff value of $189 \%$, the specificity of percentage ME to detect CA patients was $100 \%$ (sensitivity: $57 \%$ ). With a cutoff value of $152 \%$, its specificity and sensitivity were respectively $80 \%$ and $90 \%$. Examples of false positive and false negative cases are pictured in Figure 4. Except for LGE extent measured with the manual technique (AUC: 0.95 (CI: 0.88-0.99), $\mathrm{p}=0.0001)$ ), other parameters presented lower AUC values than percentage ME: post contrast myocardial T1 (AUC: 0.84 (CI: 0.68-0.99; p=0.001)), LGE extent (5SD) (AUC: 0.83 (CI: 0.76-0.97; p=0.0001)), MCF (AUC=0.70 (CI: 0.57-0.88; $\mathrm{p}=0.05)$ ) and LV mass (AUC: 0.69 (CI: 0.53-0.85; p=0.08)). The lowest values of AUC were obtained for pre 
contrast SNR (AUC=0.54 (CI: 0.37-0.71; p=0.09)), post contrast SNR (AUC=0.60 (CI: 0.440.77; $\mathrm{p}=0.09)$, pre contrast $\mathrm{CNR}(\mathrm{AUC}=0.52(\mathrm{CI}: 0.35-0.69 ; \mathrm{p}=0.09)$ and post contrast $\mathrm{CNR}$ (AUC=0.43 (CI: 0.25-0.60; p=0.09). These results are reported in Figure 3.

\section{DISCUSSION}

In this study, we report that measuring the relative myocardial enhancement (percentage ME) using pre and post contrast cine images may be of interest to distinguish CA from other causes of myocardial hypertrophy and may provide additional diagnostic confidence in everyday clinical practice.

Although morphology, function and enhancement patterns on CMR play an essential role in the assessment of CA, quantitative imaging biomarkers are relevant in order to [25]: i) define additional diagnostic criteria, particularly useful in case of equivocal CMR; ii) quantify the amyloid burden, a major prognostic marker of $\mathrm{CA}$; and iii) establish tools to assess therapeutic response. Parametric imaging, including T1 mapping techniques, increases diagnostic accuracy for CA [11,12], may distinguish ATTR from AL [11], and can quantify the amyloid burden [26]. However, these sequences remain unavailable in some centers (especially non-reference CMR centers), normal myocardial $\mathrm{T} 1$ values vary between sequences from different constructors and are influenced by physiological parameters [14,15]. Quantifying LGE extent could potentially provide information to characterize the type of amyloidosis and evaluate prognosis [27]. Nevertheless, LGE quantification is delicate and no consensus in the CMR community exists for the method to use. Although differentiating areas of LGE from non-enhancing myocardium may be obvious in cases of focal enhancement such as myocardial infarction, it becomes complex in a situation of diffuse enhancement such as in CA, and distinguishing areas of LGE can be tedious, especially when nulling of the myocardium is hard to achieve. In our experience, 
quantification of LGE extent using 2 separate methods demonstrated significantly different results in CA patients, for which LGE was often diffuse and hazy. On the other hand, similar results were found in $\mathrm{CH}$ patients, where LGE exhibited more frequently a patchy, nodular pattern. Recently, Arenja et al. have introduced a new quantitative diagnostic index, the MCF, to identify CA. This functional index, free from myocardial content characterization, presented a high diagnostic accuracy for AL amyloidosis and did not require gadolinium administration, making this metric of potential use in the assessment of LVH [22].

In our study, we report that the percentage ME measured on cine sequences was increased in CA patients compared to $\mathrm{CH}$ patients and control subjects. We also report that the percentage ME presented a higher sensitivity and specificity to detect CA than other biomarkers such as LGE extent (semi-automatic method), MCF or post contrast T1. The increase of percentage ME can be explained by the accumulation of gadolinium within the abnormally enlarged extra-cellular volume of CA patients, leading to a shortening of myocardial T1 - and therefore, an increase in SI. According to our results, obtained from a cine sequence performed 5 minutes after administration of a common dose of gadolinium, we can simply retain that an "amyloid" heart presents a relative enhancement of about $200 \%$ whereas a "normal" heart presents an enhancement of about $100 \%$. The percentage ME was easy to measure and presented a good intra and inter-examiner reproducibility. Furthermore, the percentage ME was positively correlated to the LGE extent and to post contrast myocardial T1. These results are important, because LGE and myocardial T1 are considered key elements of diagnosis on CMR. Interestingly, these correlations could indicate that the percentage ME could reflect (at least partially) the amyloid burden, an important prognostic index. Of course, because of our limited number of patients, the association between percentage ME and outcomes was not studied. Similarly, patient samples were too small to detect a difference in percentage ME between ATTR and AL patients. 
Other parameters frequently used in radiology to assess the signal, the SNR and the CNR of the myocardium before and after contrast administration, were obtained from cine images. These parameters did not achieve to distinguish CA patients from patients of other groups. For the SNR, the absence of difference may be explained by the presence of myocardial edema in CA, which has recently been advanced [28]. Myocardial edema would result in an increase of myocardial $\mathrm{T} 2$, balancing out the increase in myocardial $\mathrm{T} 1$ due to amyloid deposition. Because b-SSFP cine sequences present a T2/T1 weighting, the association of these two pathological processes, both increasing the $\mathrm{T} 1$ and the $\mathrm{T} 2$ of the myocardium, could in end not have any effect on the myocardial signal. Concerning the CNR, the absence of difference between groups could be explained by the fact that, at 5 minutes after gadolinium administration, time at which post contrast cine sequences were acquired, the typical "wash-out" of gadolinium from the blood pool may not have had time to happen yet. Suspecting CA using b-SSFP sequences may provide some advantages in clinical practice. First, in contrast to mapping sequence, b-SSFP sequences are commonly available and widely used in CMR centers. Second, it is relatively common to acquire pre and post contrast SSFP sequences during cardiac MRI scans, for instance to rapidly assess cardiac anatomy using pre contrast cine sequence and then to calculate the LVEF from post contrast contiguous SA cine sequences in order to shorten study time [29]. Third, the calculation of percentage ME that we proposed is easy to measure and can be obtained as soon as 5 minutes after contrast administration. Interestingly, this short delay was recently reported to provide the best diagnosis performance to detect CA with computed tomography (CT) [30]. Quickly obtaining additional information, before the usual 10 minutes necessary for LGE imaging, can be useful in cases of premature termination of the examination due to patient discomfort, allergic reaction to contrast or claustrophobia. Fourth, percentage ME is a ratio and is therefore independent of sequences parameters from different manufacturers. Lastly, our 
index was calculated from SA images but one can hypothesize that other acquisition planes, such as four-chambers or two-chambers planes, could be used to calculate the relative myocardial enhancement, extending the condition of its use.

Nevertheless, percentage ME presents some disadvantages. Our index requires gadolinium administration, which use can be limited in case of end-stage renal disease, often associated with amyloidosis. Pre and post contrast cine sequences parameters must be identical in order to measure the percentage ME. This parameter may be influenced by gadolinium dose and creatinine levels.

Several limitations of our study have to be underlined. First, we presented a limited number of patients, which may impact our results. Second, histological confirmation of CA was not available for patients with TTR amyloidosis. In our center we do not systematically performed EMB because it is an invasive procedure and because a positive 99mTc-HMDP scintigraphy is highly evocative of TTR amyloidosis [21]. Third, post contrast myocardial T1 values were obtained from the TI scout sequence (look-locker sequence), and not from more recent mapping sequence techniques. Nonetheless, T1 values obtained from look-locker sequences have shown good agreement with other methods [31]. However, pre contrast myocardial T1 and myocardial ECV were not available. Finally, our derivative cohort did not include cases of inflammatory cardiomyopathies such as cardiac sarcoidosis or myocarditis, which can sometimes be difficult to differentiate from cardiac amyloidosis.

\section{CONCLUSIONS}

In conclusion, we report that percentage ME can be calculated from routine pre and post contrast cine-MRI sequences and may be used as an additional parameter to suspect CA in patients with myocardial hypertrophy. 


\section{REFERENCES}

1. Elliott P, Andersson B, Arbustini E, et al. Classification of the cardiomyopathies: A position statement from the european society of cardiology working group on myocardial and pericardial diseases. Eur Heart J. 2008;29(2):270-276.

2. Damy T, Costes B, Hagège AA, et al. Prevalence and clinical phenotype of hereditary transthyretin amyloid cardiomyopathy in patients with increased left ventricular wall thickness. Eur Heart J. 2016;37(23)1826-1834.

3. Gertz MA, Dispenzieri A, Sher T. Pathophysiology and treatment of cardiac amyloidosis. Nat Rev Cardiol. 2015;12(2):91-102.

4. Syed IS, Glockner JF, Feng D, et al. Role of Cardiac Magnetic Resonance Imaging in the Detection of Cardiac Amyloidosis. JACC Cardiovasc Imaging. 2010;3(2):155-164.

5. Fontana M, Chung R, Hawkins PN, Moon JC. Cardiovascular magnetic resonance for amyloidosis. Heart Fail Rev. 2015;20(2):133-144.

6. Chawla D, Cheema O, Jan MF, Tajik AJ. Transthyretin amyloidosis in a septuagenarian masquerading as hypertrophic cardiomyopathy: the importance of multimodality imaging. Eur Heart J Cardiovasc Imaging. December 2017. doi:10.1093/ehjci/jex329.

7. Philippakis AA, Falk RH. Cardiac amyloidosis mimicking hypertrophic cardiomyopathy with obstruction: Treatment with disopyramide. Circulation. 2012;125(14):1821-1824.

8. Maceira AM, Joshi J, Prasad SK, et al. Cardiovascular magnetic resonance in cardiac amyloidosis. Circulation. 2005;111(2):186-193.

9. Kristen A V., Brokbals E, Aus Dem Siepen F, et al. Cardiac Amyloid Load A Prognostic and Predictive Biomarker in Patients with Light-Chain Amyloidosis. J Am Coll Cardiol. 2016;68(1):13-24. 
10. Maceira AM, Prasad SK, Hawkins PN, Roughton M, Pennell DJ. Cardiovascular magnetic resonance and prognosis in cardiac amyloidosis. J Cardiovasc Magn Reson. 2008;10(1):54.

11. Fontana M, Banypersad SM, Treibel TA, et al. Native T1 mapping in transthyretin amyloidosis. JACC Cardiovasc Imaging. 2014;7(2):157-165.

12. Karamitsos TD, Piechnik SK, Banypersad SM, et al. Noncontrast T1 mapping for the diagnosis of cardiac amyloidosis. JACC Cardiovasc Imaging. 2013;6(4):488-497.

13. Sado DM, Flett AS, Banypersad SM, et al. Cardiovascular magnetic resonance measurement of myocardial extracellular volume in health and disease. Heart. 2012;98(19):1436-1441.

14. Kellman P, Hansen MS. T1-mapping in the heart: accuracy and precision. $J$ Cardiovasc Magn Reson. 2014;16(2):1-20.

15. Piechnik SK, Ferreira VM, Lewandowski AJ, et al. Normal variation of magnetic resonance T1 relaxation times in the human population at $1.5 \mathrm{~T}$ using ShMOLLI. $J$ Cardiovasc Magn Reson. 2013;15(1):1.

16. Kramer CM, Barkhausen J, Flamm SD, Kim RJ, Nagel E, Society for Cardiovascular Magnetic Resonance Board of Trustees Task Force on Standardized P. Standardized cardiovascular magnetic resonance (CMR) protocols 2013 update. J Cardiovasc Magn Reson. 2013;15:91.

17. Chavhan GB, Babyn PS, Jankharia BG, Cheng H-LM, Shroff MM. Steady-state MR imaging sequences: physics, classification, and clinical applications. Radiographics. 2008;28(4):1147-1160.

18. Deux JF, Maatouk M, Lim P, et al. Acute myocarditis: Diagnostic value of contrastenhanced cine steady-state free precession MRI sequences. Am J Roentgenol. 2011;197(5):1081-1087. 
19. Codreanu A, Djaballah W, Angioi M, et al. Detection of myocarditis by contrastenhanced MRI in patients presenting with acute coronary syndrome but no coronary stenosis. J Magn Reson Imaging. 2007;25(5):957-964.

20. Nordlund D, Kanski M, Jablonowski R, et al. Experimental validation of contrastenhanced SSFP cine CMR for quantification of myocardium at risk in acute myocardial infarction. J Cardiovasc Magn Reson. 2017;19(1):12.

21. Gillmore JD, Maurer MS, Falk RH, et al. Nonbiopsy diagnosis of cardiac transthyretin amyloidosis. Circulation. 2016;133(24):2404-2412.

22. Arenja N, Fritz T, Andre F, et al. Myocardial contraction fraction derived from cardiovascular magnetic resonance cine images-reference values and performance in patients with heart failure and left ventricular hypertrophy. Eur Heart J Cardiovasc Imaging. 2017;18(12):1414-1422.

23. Burt JR, Zimmerman SL, Kamel IR, Halushka M, Bluemke DA. Myocardial T1 mapping: techniques and potential applications. Radiographics. 2014;34(2):377-395.

24. Bondarenko O, Beek AM, Hofman MBM, et al. Standardizing the definition of hyperenhancement in the quantitative assessment of infarct size and myocardial viability using delayed contrast-enhanced CMR. J Cardiovasc Magn Reson. 2005;7(2):481-485.

25. Falk RH, Quarta CC, Dorbala S. How to image cardiac amyloidosis. Circ Cardiovasc Imaging. 2014;7(3):552-562.

26. Banypersad SM, Sado DM, Flett AS, et al. Quantification of myocardial extracellular volume fraction in systemic AL amyloidosis: An equilibrium contrast cardiovascular magnetic resonance study. Circ Cardiovasc Imaging. 2013;6(1):34-39.

27. Fontana M, Pica S, Reant P, et al. Prognostic value of late gadolinium enhancement cardiovascular magnetic resonance in cardiac amyloidosis. Circulation. 
2015;132(16):1570-1579.

28. Fontana M, Banypersad SM, Treibel TA, et al. Differential Myocyte Responses in Patients with Cardiac Transthyretin Amyloidosis and Light-Chain Amyloidosis: A Cardiac MR Imaging Study. Radiology. 2015;277(2):388-397.

29. Lasalarie JC, Serfaty JM, Carre C, et al. Accuracy of contrast-enhanced cine-MR sequences in the assessment of left ventricular function: Comparison with precontrast cine-MR sequences. Results of a bicentric study. Eur Radiol. 2007;17(11):2838-2844.

30. Rosmini S, Treibel TA, Bandula S, et al. Cardiac computed tomography for the detection of cardiac amyloidosis. J Cardiovasc Comput Tomogr. 2017;11(2)155-156.

31. Nacif MS, Turkbey EB, Gai N, et al. Myocardial T1 mapping with MRI: comparison of look-locker and MOLLI sequences. J Magn Reson Imaging. 2011;34(6):1367-1373. 


\section{Figure Legends}

Figure 1. Examples of CMR images for patients from each group.

Examples of CMR of one patient with $\mathrm{CA}$ (first column), one patient with $\mathrm{CH}$ (second column) and one control patient (third column). End-diastolic pre contrast (Panels A, B, and C) and post contrast (Panels D, E, and F) cine b-SSFP images acquired at the mid-ventricular level. Note the increase of myocardial SI on post contrast cine image in CA patient (Panel D) in comparison to other patients. Percentage ME was higher in patients with CA (200\%) than patients with $\mathrm{CH}(122 \%)$ and control patients (104\%). LGE imaging evidences typical subendocardial circumferential enhancement in patients with CA (Panel G) and focal enhancement at the junction between right and left ventricles in patients with $\mathrm{CH}(\mathrm{Panel} \mathrm{H})$. No LGE was detected in control patients (Panel I).

Figure 2. Correlations of Percentage ME with LGE extent and post contrast T1.

Percentage ME was significantly correlated with LGE extent measured with the manual method (Rho Spearman coefficient $=0.75$; $\mathrm{p}=0.0001$; panel A) and with post contrast myocardial T1 (Rho Spearman coefficient=-0.61; $\mathrm{p}=0.0001$; panel B).

Figure 3. Receiver-operating characteristic (ROC) curves.

On this graph are represented ROC curves for Percentage ME (blue line; AUC: 0.92), LGE extent measured by semi-automatic method (AUC: 0.83 , red line), LGE extent measured by manual method ( $\mathrm{AUC}=0.95$, green line $), \mathrm{MCF}(\mathrm{AUC}=0.70$, orange line $)$ and post contrast myocardial T1 (AUC: 0.84, yellow line).

Figure 4. Examples of false positive and false negative cases. 
Example of 2 patients with LVH, a false positive and a false negative case, using a cut-off value of $152 \%$ for Percentage ME. On the first line are presented short-axis mid-LV pre contrast cine (A), post contrast cine (B) and LGE (C) images for a patient with confirmed hypertrophic cardiomyopathy. In this case, the Percentage ME was $186 \%$. On the second line are presented short-axis mid-LV pre contrast cine (D), post contrast cine (E) and LGE (F) images for a patient with confirmed cardiac amyloidosis. In this case, the Percentage ME was $124 \%$. 


\section{Table Legends}

\section{Table 1. Population characteristics}

$\mathrm{CA}=$ cardiac amyloidosis; $\mathrm{CH}=$ cardiac hypertrophy; GLS = Global Longitudinal Strain; IVS = interventricular septum thickness; $\mathrm{LVEF}=$ left ventricular ejection fraction; N/A = not applicable;

Data are given as mean \pm standard deviation $(\mathrm{SD})$ or median (IQR).

*Measured on transthoracic echocardiography.

**P-value for CA vs. $\mathrm{CH}$ patients.

$* * * \mathrm{P}-\mathrm{value}$ for CA vs. control patients.

${ }^{\$} \mathrm{p}<0.05$ for both $\mathrm{CA}$ vs. control patients and $\mathrm{CH}$ vs. control patients.

${ }^{€}$ No significant difference between $\mathrm{CH}$ and control patients.

\section{Table 2. Comparison of CMR parameters between groups}

$\mathrm{CA}=$ cardiac amyloidosis; $\mathrm{CH}=$ cardiac hypertrophy; $\mathrm{CNR}=$ contrast-to-noise ratio; IVS = interventricular septum; $\mathrm{LVEF}=$ left ventricular ejection fraction; MCF: myocardial contraction fraction; Percentage ME = percentage of myocardial enhancement; SNR = signalto-noise ratio. Data are given as mean \pm standard deviation $(\mathrm{SD})$ or median (IQR).

*Measured on CMR.

**P-value for CA vs. $\mathrm{CH}$ patients.

***P-value for CA vs. control patients.

${ }^{\$} \mathrm{p}<0.05$ for both CA vs. control patients and $\mathrm{CH}$ vs. control patients.

${ }^{€}$ No significant difference between $\mathrm{CH}$ and control patients. 


\section{Additional Files Legends}

\section{*Additional file 1}

Title: Bland-Altman plot for intra-examiner reproducibility of percentage ME.

Description: Horizontal axis represents the mean of the 2 measures. Vertical axis represents the difference between the 2 measures. Most measures are located in between limits of agreement, demonstrating reasonable reproducibility.

\section{*Additional file 2}

Title: Bland-Altman plot for inter-examiner reproducibility of percentage ME.

Description: Horizontal axis represents the mean of the 2 measures. Vertical axis represents the difference between the 2 measures. Most measures are located in between limits of agreement, demonstrating reasonable reproducibility.

\section{*Additional file 3}

Title: Examples of LGE quantification.

Description: Examples of LGE imaging (PSIR) for a patient with hypertrophic cardiomyopathy ( $1^{\text {st }}$ line), and 2 patients with cardiac amyloidosis ( $2^{\text {nd }}$ and $3^{\text {rd }}$ lines). Quantification of LGE extent by a semi-automatic method (threshold +5SD) is shown in the $2^{\text {nd }}$ column and by manual method in the $3^{\text {rd }}$ column. Similar results of LGE extent between methods were observed in situations of focal LGE (panels B, C). When LGE was more diffuse, discordant results between the 2 methods were often recorded (panels E, F and H, I). 
$A$

$\begin{array}{lll}B & C\end{array}$

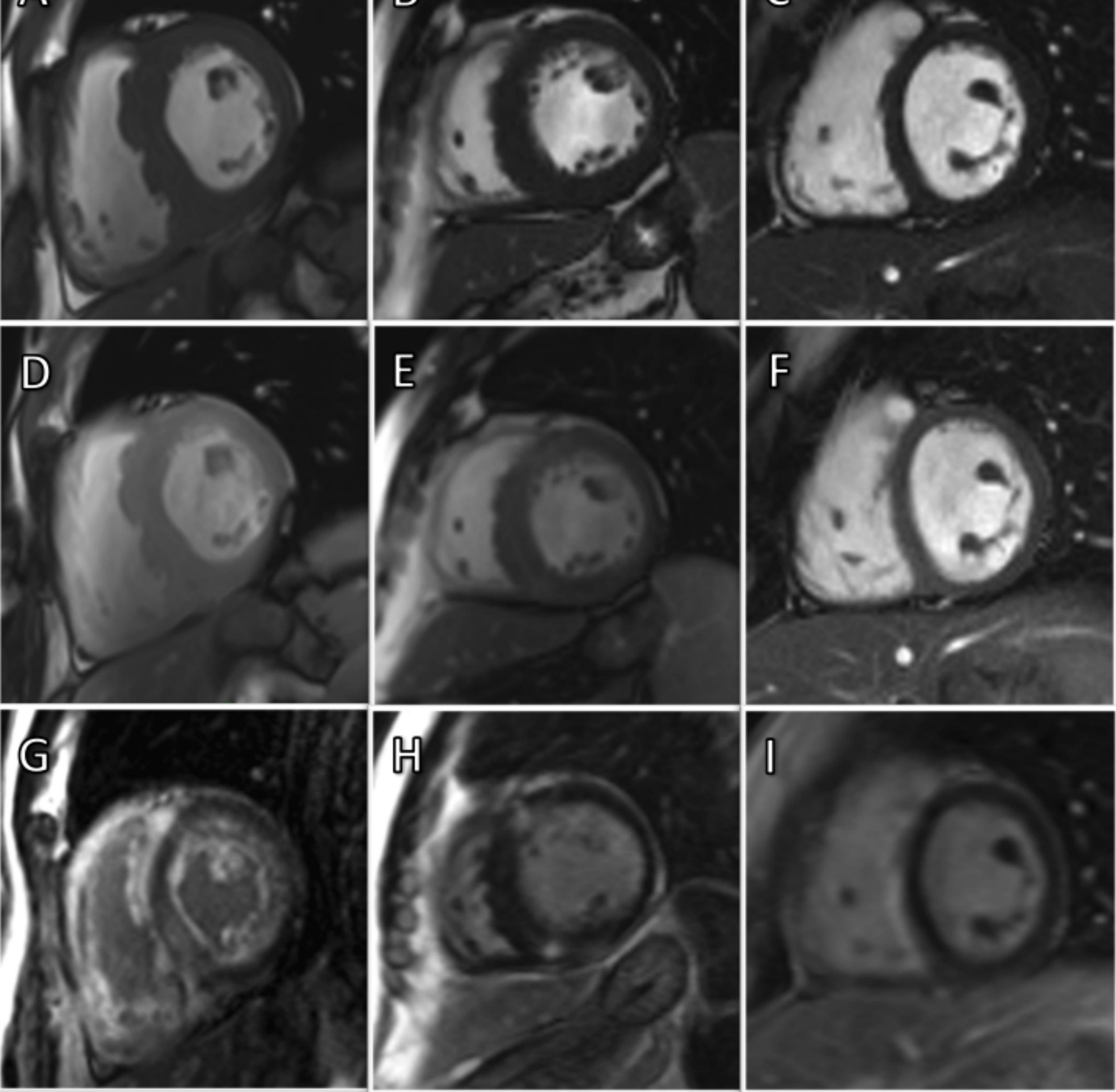




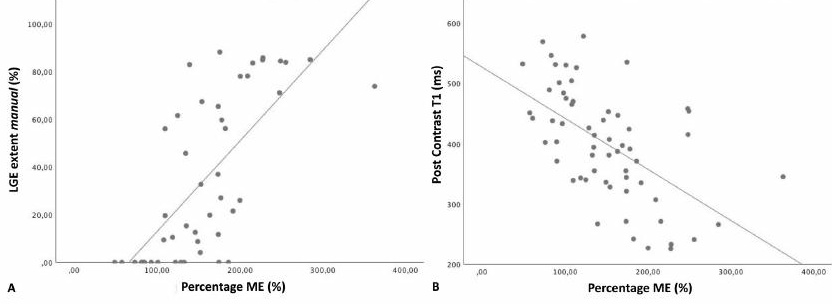




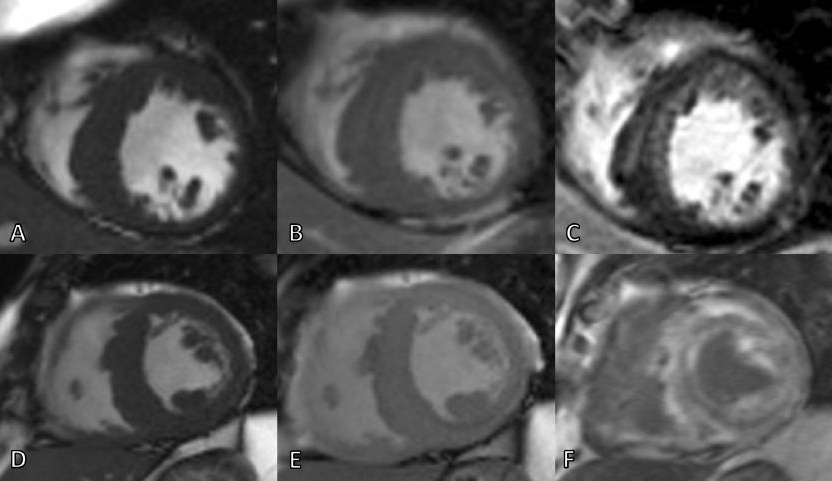


Table 1. Population characteristics

\begin{tabular}{lccc||rc}
\hline & CA $(\mathrm{n}=21)$ & CH $(\mathrm{n}=25)$ & $p^{* *}$ & Control $(\mathrm{n}=20)$ & $p^{* * *}$ \\
\hline Age (years) & $70 \pm 14$ & $62 \pm 14$ & 0.09 & $49 \pm 18$ & $0.001^{\$}$ \\
Male $(\%)$ & $17(81)$ & $16(64)$ & 0.3 & $12(60)$ & 0.4 \\
NT-pro BNP $(\mathrm{pg} / \mathrm{mL})$ & $1849(564-5215)$ & $331(55-1245)$ & 0.004 & $217(28-390)$ & $0.001^{€}$ \\
Troponin T $(\mathrm{pg} / \mathrm{mL})$ & $52(14-112)$ & $18(8-24)$ & 0.04 & $12(8-14)$ & $0.01^{€}$ \\
Creatinine $(\mu \mathrm{mol} / \mathrm{L})$ & $109(81-143)$ & $89(64-109)$ & 0.04 & $85(61-193)$ & $0.04^{€}$ \\
IVS $(\mathrm{mm}) *$ & $17 \pm 5$ & $18 \pm 9$ & 0.6 & $9 \pm 2$ & $0.001^{\$}$ \\
LVEF $(\%) *$ & $53 \pm 15$ & $53 \pm 14$ & 0.9 & $62 \pm 6$ & 0.9 \\
GLS $(-\%) *$ & $11 \pm 5$ & $15 \pm 5$ & 0.8 & N/A & N/A \\
\hline
\end{tabular}


Table 2. Comparison of CMR parameters between groups

\begin{tabular}{|c|c|c|c|c|c|}
\hline & $\overline{\mathbf{C A}}$ & $\mathbf{C H}$ & $p^{* *}$ & Control & $p^{* * * *}$ \\
\hline IVS thickness $(\mathrm{mm})^{*}$ & $19 \pm 4$ & $16 \pm 3$ & 0.008 & $9 \pm 2$ & $0.0001^{\$}$ \\
\hline LVEDV (mL/m²) & $78 \pm 31$ & $82 \pm 35$ & 0.8 & $70 \pm 14$ & 0.8 \\
\hline LVESV (mL/m²) & $42 \pm 29$ & $41 \pm 33$ & 0.6 & $28 \pm 8$ & 0.6 \\
\hline $\mathbf{L V} \operatorname{mass}\left(\mathrm{g} / \mathrm{m}^{2}\right)$ & $107 \pm 39$ & $90 \pm 34$ & 0.008 & $65 \pm 11$ & $0.03^{\$}$ \\
\hline LVEF $(\%)^{*}$ & $50 \pm 17$ & $53 \pm 15$ & 0.1 & $61 \pm 9$ & 0.1 \\
\hline Percentage ME (\%) & $200(174-238)$ & $122(88-151)$ & 0.0001 & $104(90-149)$ & $0.0001^{€}$ \\
\hline MCF (\%) & $33(26-52)$ & $54(44-64)$ & 0.007 & $79(72-96)$ & $0.0001^{\$}$ \\
\hline LGE extent ${ }_{S S D}(\%)$ & $43 \pm 26$ & $11 \pm 15$ & 0.0001 & $0 \pm 0$ & $0.0001^{\$}$ \\
\hline LGE extent manиа $_{\text {(\%) }}$ & $65 \pm 22$ & $11 \pm 17$ & 0.0001 & $0 \pm 0$ & $0.0001^{\$}$ \\
\hline Post contrast T1 (ms) & $271(242-340)$ & $439(386-482)$ & 0.0001 & $411(390-499)$ & $0.0001^{€}$ \\
\hline $\mathrm{SNR}_{\text {myo IV- }}$ & $35(22-115)$ & $31(16-116)$ & 0.5 & $29(24-41)$ & 0.7 \\
\hline $\mathrm{SNR}_{\text {myo IV+ }}$ & $113(55-205)$ & $68(37-148)$ & 0.3 & $63(43-130)$ & 0.3 \\
\hline $\mathrm{CNR}_{\text {myo IV- }}$ & $190(92-397)$ & $158(86-471)$ & 0.7 & $144(112-235)$ & 0.9 \\
\hline $\mathrm{CNR}_{\text {myo IV+ }}$ & $70(35-223)$ & $120(60-187)$ & 0.3 & $135(75-292)$ & 0.2 \\
\hline
\end{tabular}

